\title{
Important considerations in the testing of high density tailings for beach profile prediction
}

\section{G.I. McPhail SLR Consulting Australia (Pty) Ltd, Australia}

M. Becerra ASMIN, Chile

S. Barrera ARCADIS, Chile

\section{Abstract}

The authors have conducted several investigations into the prediction of the beach profile of high density tailings and have found that there are a number of important considerations that need to be taken into account over and above the reliability of the beach profile prediction methodology. These considerations relate to the chemistry of the slurry and specifically the influence of $\mathrm{pH}$ and flocculation, both of which have a significant influence on the rheology of the slurry at the discharge point. A number of examples are discussed and a potential testing protocol that will assist in assessing the influence of chemistry ahead of the beach profile predictions is presented. A methodology for assimilating the results of the testing into the likely field situation is discussed and suggestions are made on application of the results in beach profile prediction. The suspected influence of residual flocculation on the stratification of the tailings slurry as it flows down the beach is also discussed.

\section{Introduction}

Beach profile prediction methodologies currently being applied to tailings slurries generally make use of the rheological properties of the slurry as determined by laboratory testing, large-scale flume testing, pilotscale beach trials and pipe loop testing. Laboratory testing mostly involves shear testing, using a vane rheometer to derive an estimate of the yield stress of the slurry and viscometer testing to derive a relationship for the flow diagram beyond the yield stress. The laboratory testing methods have their limitations in terms of settling out of slurries, a particular problem in slurries of high sand content, and/or slippage during the tests, and focus on the derivation of rheological parameters only; i.e. they do not attempt to simulate the processes occurring during beaching itself. The tests are also of very limited sample size. However, they have an important advantage in that they are relatively inexpensive and quick, which means that tests can be repeated and a range of samples can be tested in a single programme.

Flume tests, pilot-scale beach trials and pipe loop tests, on the other hand, are conducted at a scale and using a methodology specifically designed to be as representative of the slurry behaviour as possible. However, they have costly equipment requirements. The size of the tailings sample and the effort required to prepare it to the required slurry density, limits the number of tests that can be conducted and therefore limits the flexibility, and ultimately the representativeness, of the testing programme. The question soon becomes one of whether the sample size and scalability of the pilot-scale results justifies the lack of flexibility and, indeed, whether this flexibility is even required.

Over the course of a number of projects requiring the prediction of the beaching characteristics of high density tailings at a range of percentage solids, the authors have observed the influence of a number of factors that affect the reliability of the estimates of the rheology of a slurry. Specifically, the interaction of reagents, flocculants and extraction chemistry ultimately determines the chemistry of the slurry and the chemistry has a strong influence on the rheology. This observation is important because it is not always possible to fully simulate the chemistry of the full-scale processing situation when doing limited scale tests. Indeed, in the case of a greenfields operation, it is impossible to fully simulate the full-scale chemistry. The authors have also observed the beaching characteristics of high sand content slurries. An obvious example 
is hydrocyclone underflow. These slurries exhibit very low yield stresses and, from pipe loop tests, appear to behave as Newtonian, yet they present beach slopes of the order of $20-30 \%$. It is therefore clear that particle size distribution also plays a very significant role in the determination of the beaching profile. These considerations are over and above the issues of sample representativeness in terms of mineralogy and particle size distribution.

A number of examples illustrating the influence of slurry chemistry, as well as a number of other considerations are set out below together with an approach that attempts to bring them into consideration in the beach profile prediction process. But first, it is of interest to note the influence of rheological test methodology.

\section{Influence of rheological test methodology}

Figure 1 shows a plot of yield stress versus percentage solids of a tailings slurry. The yield stresses have been estimated three ways, namely:

- using a vane rheometer

- through pipe loop testing

- by means of beach trials based on the stream power-entropy methodology (McPhail, 1995, 2008).

None of the methods specifically determines the yield stress, which by definition, occurs at zero shear rate. Indeed, this is an issue with all rheological testing methods. In the case of the vane rheometer, the shear rate is indeterminable, so the measured yield stress is an estimate. In the case of the pipe loop tests and the beach trials, both of which involved the preparation and application of $3 \mathrm{~m}^{3}$ of slurry, the yield stress has had to be estimated by extrapolating back to the yield stress axis on the flow diagram.

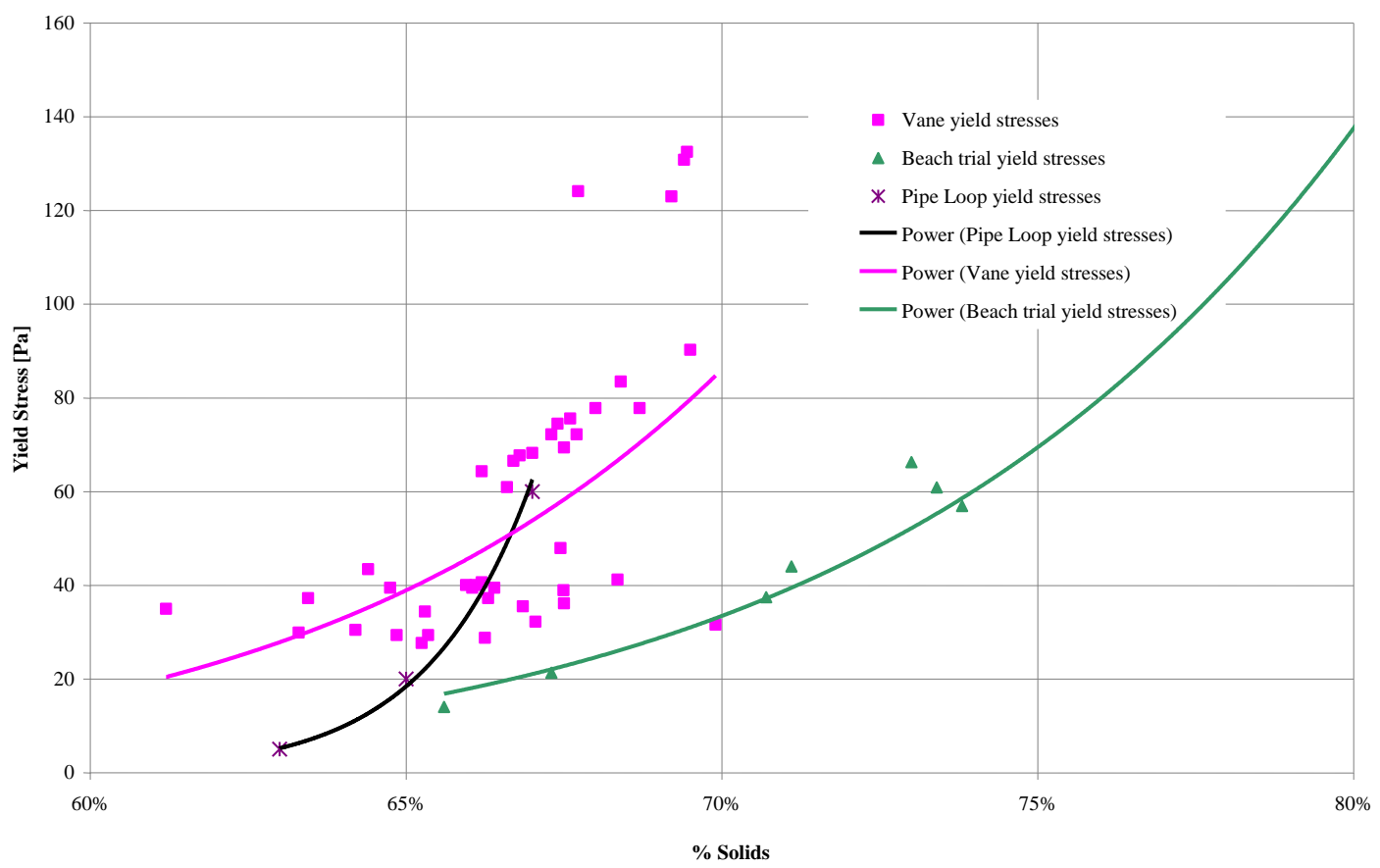

Figure 1 Yield stress versus percentage solids for various test methods on the same tailings

It is evident from Figure 1 that there is considerable variability in the estimated yield stress between the methods. In this case, the vane rheometer and the beach trials have been conducted on exactly the same batch of slurry, but the pipe loop tests have been conducted on a separate batch of slurry. The tailings solids are from one sample set and therefore it is likely that differences in the preparation of the batches are also playing a role in the scatter. Since the tailings are from the same set of samples, which have been ground and blended, the differences are most likely related to slurry chemistry. 
The influence of $\mathrm{pH}$ on the rheology of a slurry is generally well appreciated, although not necessarily fully understood by thickener designers, and it is not always taken into consideration during testing for the purpose of beach profile estimation. Figure 2 shows a plot of yield stress versus percentage solids from vane testing on a tailings slurry. It is evident that there are two points that plot well below the trend line. These two points were conducted on a slurry at a $\mathrm{pH}$ of 8 , whereas the slurries on the trend line were all conducted at a $\mathrm{pH}$ of 9.

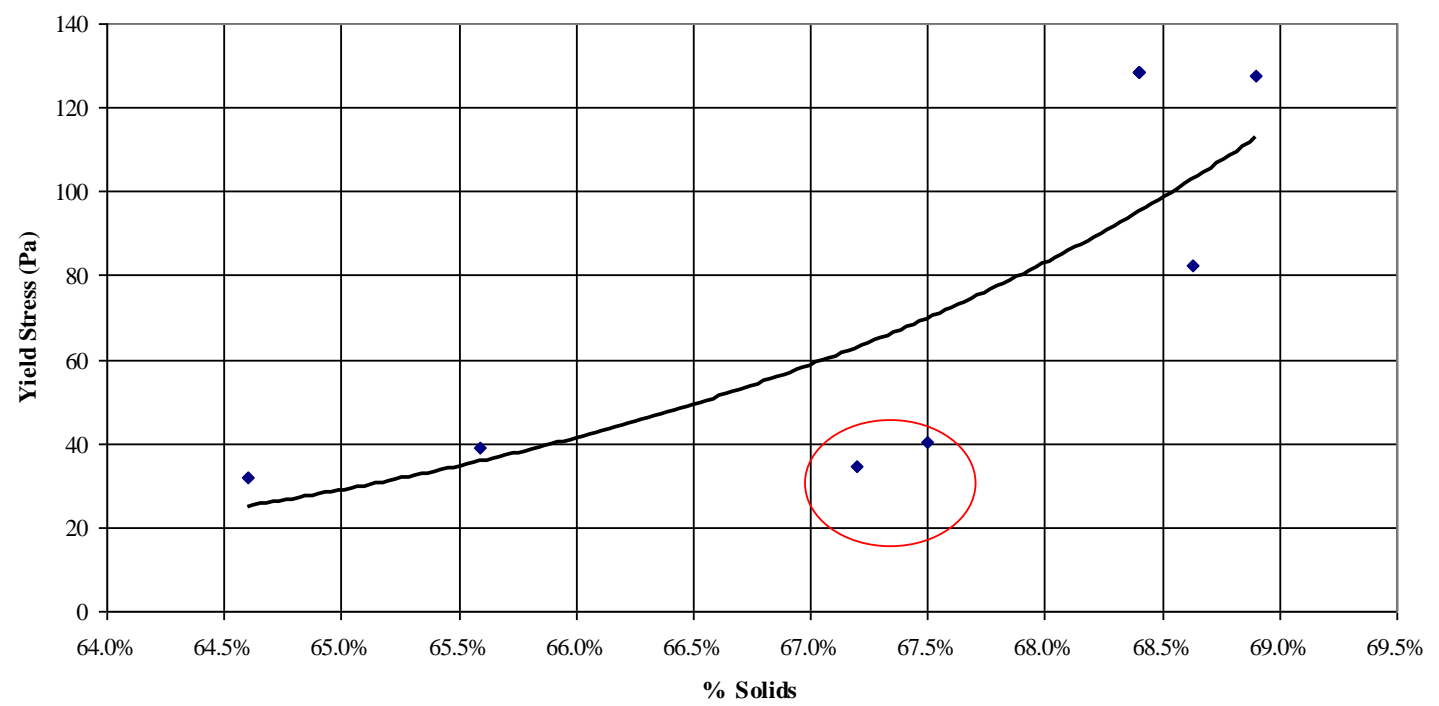

Figure 2 Yield stress versus percentage solids from vane testing

It is evident from Figure 2 that, in this case, the $\mathrm{pH}$ difference could result in a $30-40 \%$ under- or overestimation of the yield stress, depending on whether neutralisation in the full-scale operation was taken into consideration in the testing programme.

\section{Influence of flocculant type and dose}

Testing of high density slurries has a strong focus on the type and dose rate of flocculant to optimise thickener performance. There are many flocculants available on the market and it is normal for thickener vendors to conduct tests on a range of flocculants to determine which brand is the most effective for the specific tailings. But if the flocculant test results are not available at the time of testing for the beach profile prediction, the testing laboratory has no other option but to generate tailings at the appropriate density, using process plant liquor or, if this is unavailable, as in the case of greenfields projects, using tap water.

If pilot-scale testing is being carried out, the laboratory generating the tailings will generally have a pilotscale thickener on site. Unless the testing is being done adjacent to an operating plant, so that large sources of process liquor are available, the slurry has to be mixed up with tap water and the $\mathrm{pH}$ adjusted. Then, in order to generate the slurry at the correct density for the testing, a flocculant is added to the slurry in the thickener. Very likely the flocculant dose will be elevated so as to minimise the sample preparation time.

Figure 3 shows a plot of yield stress versus per cent solids measured, using a vane rheometer on the same slurry but with and without flocculant dosage. The samples are taken directly from the base of the thickener and are therefore unsheared. 


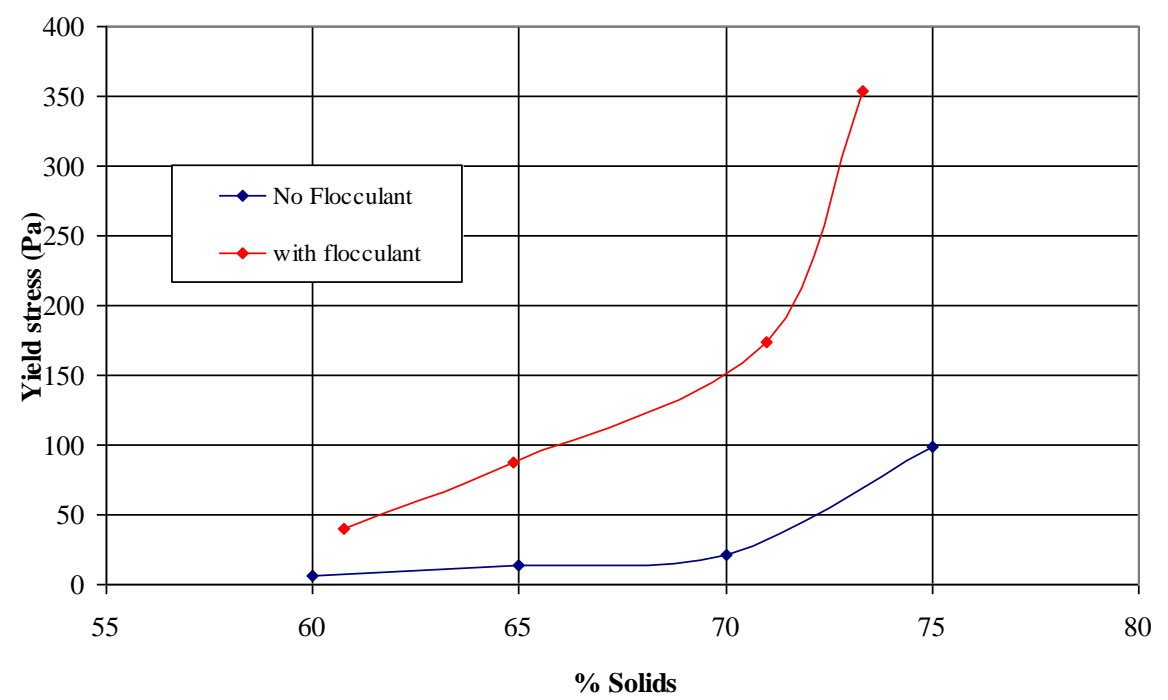

Figure 3 Yield stress versus percentage solids with and without flocculant

It is evident from Figure 3 that flocculant has a very significant effect on the yield stress of the unsheared slurry, potentially increasing it by a factor of three or more.

\section{$5 \quad$ Influence of shear thinning}

Just as the addition of flocculant increases the yield stress, so shearing of the slurry breaks down the flocc and induces shear thinning with a concomitant reduction in yield stress. Indeed it is common to build shear thinning into the design of a high density slurry transport system.

Figure 4 indicates the effect on the yield stress, at a range of per cent solids, of shear thinning induced by longer agitation, and therefore longer shearing.

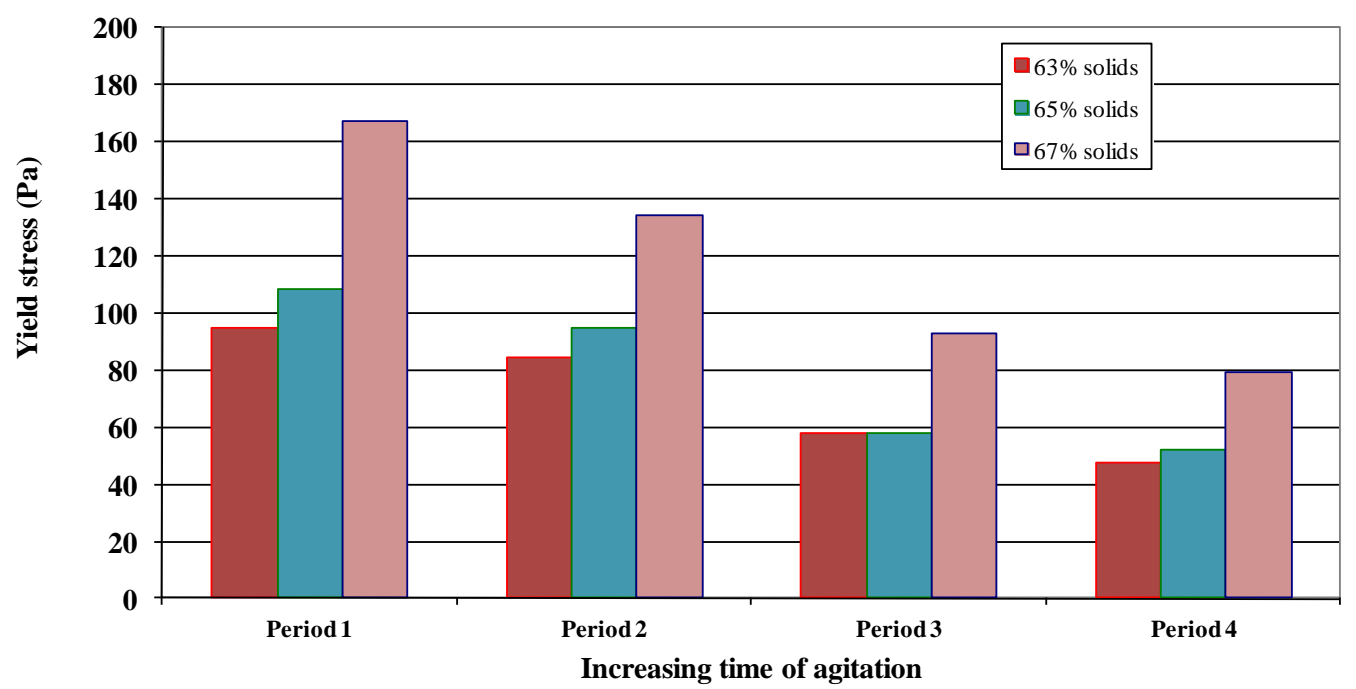

Figure 4 Influence of agitation-induced shear thinning on the yield stress

It is evident from Figure 4 that the yield stress reduced by more than $50 \%$ in the case of the slurry tested. More important is the fact that, in order to estimate the yield stress on discharge onto the beach in the fullscale operation, it is necessary to estimate the degree of shearing up to the time of discharge. Conversely, if a higher yield stress is required it is necessary to estimate the extent to which it is practical to minimise the degree of shear thinning up to the time of discharge.

Because these considerations have a very important impact on the testing of the slurry rheology, it is evident that when testing the slurry it will be important to either: 
- ensure that a range of slurries are tested from fully flocculated to fully shear thinned, as this will facilitate estimation of the effect of shear thinning in the full-scale operation, or

- give consideration to the likely degree of shear thinning in the full-scale operation so as to try to ensure that the sample is similarly shear thinned prior to testing.

\section{Influence of shear thickening}

Tailings that possess a clay fraction such as montmorlilonite may exhibit shear thickening behaviour. Figure 5 shows the change in yield stress of a shear thickening slurry at $66 \%$ solids with increasing agitation duration. It is evident from Figure 5 that in the case of the slurry tested; the yield stress could more than double depending on the duration of shearing.

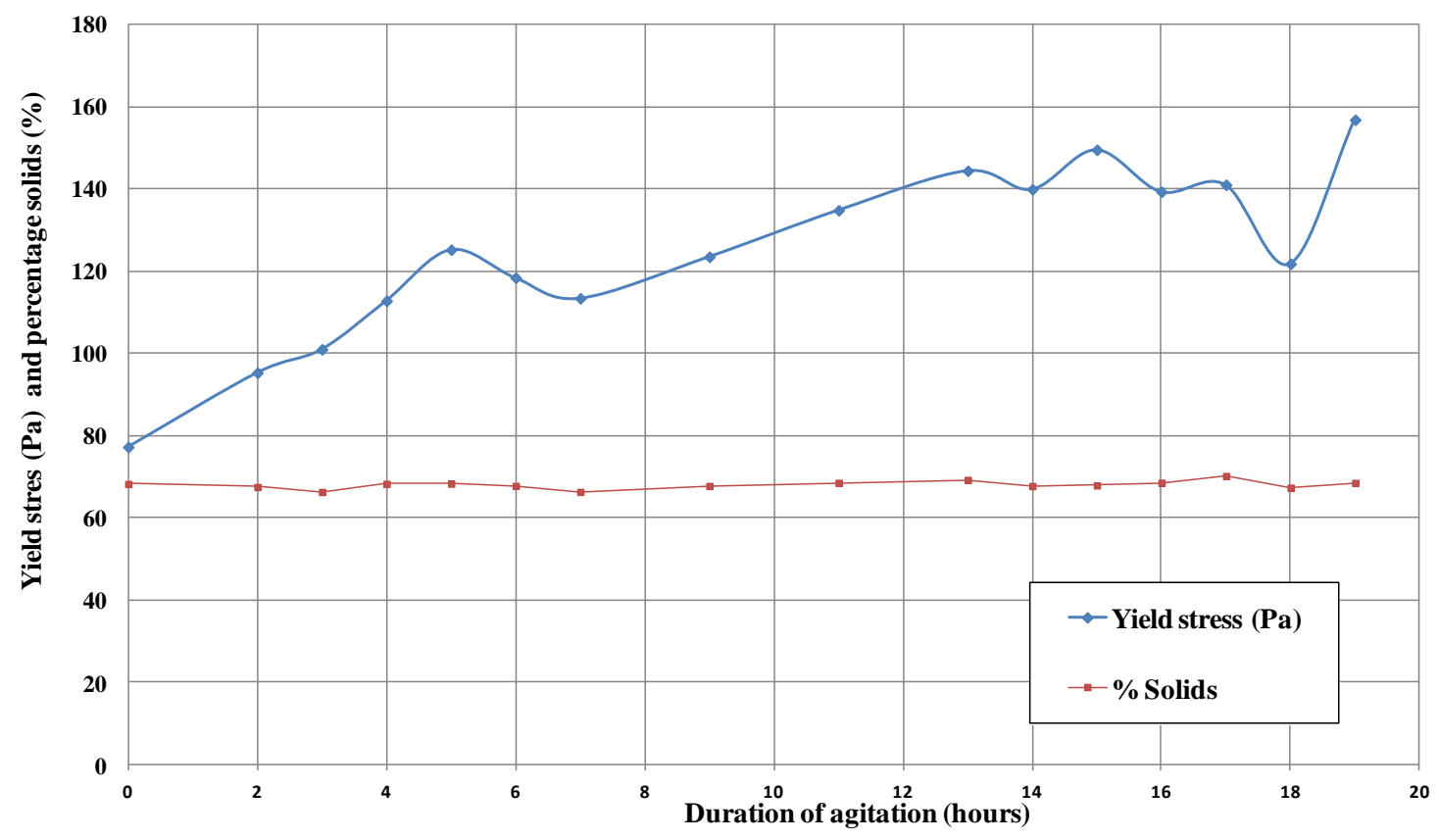

Figure 5 Influence of agitation-induced shear thickening on the yield stress

As in the case of a shear thinning slurry it is essential to give consideration to the likely degree of shear thickening at the point of discharge in the full-scale operation and then ensure that laboratory and smallscale beaching trials provide data that will cover the range of anticipated shear thickening.

\section{Influence of stratification of the slurry stream}

Tailings slurries at conventional consistencies of $35-50 \%$ solids commonly undergo gravitational segregation of the tailings during flow down the beach. For these tailings it is generally observed that the coarser particles in the slurry settle out early on the beach causing the deposited tailings to become finer with distance down the beach.

Pinto and Barrera (2002) present beach slope data for copper tailings from seven copper mines where there are variations in particle specific gravity, particle size distribution and slurry per cent solids. It is shown that even for tailings of similar specific gravity and similar density there can be significant differences in the beach slope of the tailings.

It is generally observed that gravitation segregation reduces as the slurry per cent solids increases. The authors have worked with tailings, which even at $57 \%$ solids, does not show significant segregation into coarse and fine tailings down the beach. However, for these tailings there is significant difference in the full-scale beach slopes as indicated in Table 1. Also indicated in Table 1 is the yield stresses applicable to each slurry based on back-analysis of the full-scale beach profile using the stream power-entropy 
methodology (McPhail, 2008; Simms et al., 2011). There is a significant difference in the estimated yield stress.

Table 1 Back-calculated full-scale yield stresses for two copper tailings slurries at $57 \%$ solids

\begin{tabular}{ccccc}
\hline $\begin{array}{c}\text { Particle Specific } \\
\text { Gravity }\end{array}$ & $\begin{array}{c}\mathrm{D}_{\mathbf{8 0}} \\
\text { (micron) }\end{array}$ & $\begin{array}{c}\mathrm{D}_{50} \\
\text { (micron) }\end{array}$ & $\begin{array}{c}\text { Average Beach } \\
\text { Slope (\%) }\end{array}$ & $\begin{array}{c}\text { Back-calculated } \\
\text { Yield Stress (Pa) }\end{array}$ \\
\hline 2.73 & 250 & 75 & 0.22 & 20 \\
2.70 & 360 & 100 & 0.60 & 80 \\
\hline
\end{tabular}

Mineralogical differences between the slurries, as well as the difference in grind, will contribute to the difference in beach slopes. However, it is the principal author's contention that based on observation of high density slurries as these flow down the beach in both small-scale flumes as well as in full-scale operations, the difference relates also to density stratification of the slurry as this flows down the beach. It is argued that the slurry flowing closest to the bed is at a higher per cent solids than the average per cent solids of the slurry and therefore possess a higher yield stress than the slurry as a whole. Moreover, it is contended that the extent of stratification is related to the degree of shear thinning and residual flocc characteristics of the flocculated slurry. Since the beach slope is determined by the rheology of the material at the bed face where the deposition of solids takes place, and given the relatively small differences in particle specific gravity and grind, it is considered likely that density stratification accounts for the majority of the differences set out in Table 1.

\section{Influence of sand content}

High sand content slurries, such as those generated when the run of mill tailings stream is split into fine and coarse particles using hydrocyclones are, in the authors' experience, generally of low to negligible yield stress. This is because cohesive forces responsible for generating yield stress in slurries are more active between fine particles. In such cases the beach profile is generally steep whatever the percentage solids of the slurry. At conventional percentage solids, the sands rapidly migrate to the base of the flow stream after discharge and the upper part of the flow stream is dominated by water flow. In high density slurries there is less separation of water and sands. As a result, most sand slurries beach steeply at $20-30 \%$. These slopes are achieved in the absence of a yield stress and the beaching behaviour appears to be dominated by viscosity.

It would seem logical therefore, that run of mill tailings that have a high sand content will have their beaching characteristics determined by both the yield stress generated by the fine fraction in the tailings, as well as by the sand fraction, which will tend to influence the apparent viscosity of the slurry.

\section{$9 \quad$ Sensitivity of beach profile predictions to the yield stress}

The net effect of the factors discussed in Sections 2 to 6 is to introduce uncertainty into the estimate of yield stress. The question is whether the range will have a significant effect on the beach profile predictions. To assess this, consider a typical tailings operating scenario:

- Annual tonnage: 10 Mtpa.

- Solids specific gravity: 2.7 .

- Slurry percentage solids: $68 \%$.

- Discharge setup: 2 spigots of $250 \mathrm{~mm}$ diameter.

- Beach length: $2,000 \mathrm{~m}$.

- Yield stress: 20-100 Pa. 
The stream power-entropy method of analysis (McPhail, 2008; Simms et al., 2011) has been used to predict the beach profiles for yield stresses of $20,40,60,80$ and $100 \mathrm{~Pa}$. To ensure that the influence on beach profile is confined to variation in the yield stress, the slurry is taken as a Bingham plastic with a constant Bingham viscosity of 0.05 Pas. In reality, the factors discussed in Sections 2 to 6 also have an influence on the apparent viscosity with the changes in viscosity most likely emulating the changes in yield stress.

Table 2 below shows the resulting average beach profiles, as well as a measure of the concavity of the profile, where the measure is calculated as the maximum vertical height difference between the predicted beach profile and a straight line profile at the average slope.

Table 2 Beach profiles for a range of yield stresses

\begin{tabular}{cccc}
\hline $\begin{array}{c}\text { Yield Stress } \\
(\mathbf{P a})\end{array}$ & $\begin{array}{c}\text { Assumed Bingham } \\
\text { Viscosity (Pas) }\end{array}$ & $\begin{array}{c}\text { Average Beach } \\
\text { Slope }(\%)\end{array}$ & $\begin{array}{c}\text { Concavity* } \\
(\mathbf{m})\end{array}$ \\
\hline 20 & 0.05 & 0.85 & 0.13 \\
40 & 0.05 & 2.06 & 0.66 \\
60 & 0.05 & 3.32 & 1.83 \\
80 & 0.05 & 4.59 & 3.55 \\
100 & 0.05 & 5.81 & 5.81 \\
\hline
\end{tabular}

* Measured as the maximum vertical height difference between the predicted beach profile and a straight line profile at the average slope.

It is evident from Table 2 that yield stress has a very significant impact on the beach profile predictions.

\section{Sensitivity of the beach profile to viscosity}

To assess the importance of viscosity on the beach profile prediction, consider the example mining situation applied in Section 9 but assume the yield stress is a constant $20 \mathrm{~Pa}$, the flow diagram linear, and the resulting Bingham viscosity to vary from 0.02-0.5 Pas. Table 3 sets out beach profile results predicted, using the stream power-entropy method (McPhail, 2008; Simms et al., 2011).

Table 3 Beach profiles for a range of yield stresses

\begin{tabular}{cccc}
\hline $\begin{array}{c}\text { Assumed Bingham } \\
\text { Viscosity (Pas) }\end{array}$ & $\begin{array}{c}\text { Yield Stress } \\
(\mathbf{P a})\end{array}$ & $\begin{array}{c}\text { Average Beach } \\
\text { Slope }(\%)\end{array}$ & $\begin{array}{c}\text { Concavity* } \\
(\mathbf{m})\end{array}$ \\
\hline 0.02 & 20 & 0.82 & 0.124 \\
0.1 & 20 & 0.88 & 0.174 \\
0.5 & 20 & 1.05 & 0.295 \\
\hline
\end{tabular}

* Measured as the maximum vertical height difference between the predicted beach profile and a straight line profile at the average slope.

The influence of the Bingham viscosity on the beach slope is shown to be relatively minor compared with the yield stress, but an important factor nonetheless.

\section{Suggested rheological testing approach}

In light of the observations set out in Sections 2 to 8, it would seem prudent, in developing and implementing a rheology testing programme for the purposes of beach profile prediction, to ensure that the following points are observed:

- As far as practically possible, the liquor used to make up the slurry samples should be representative of the process water liquor. On greenfields projects, samples of the liquor may become available during the metallurgical pilot testing and this liquor should be collected and preserved for use in the flocculation settling tests and then used directly in the rheological testing. 
- The rheological testing should be conducted on samples that cover the range of flocculant types and dosage rates being considered for the tailings. This means that it is preferable for the testing to take place simultaneously, with the settling tests conducted as part of the thickener assessments.

- Laboratory testing should be conducted on samples of varying degrees of shear thinning. Use can be made of a slump test to assess the degree of shear thinning between consecutively sheared samples. Samples should be repeatedly sheared, tested and a slump test conducted until the slump tests show constant values. The sample will then be fully sheared. When conducting vane rheometer testing in the laboratory, it is further recommended that the bucket rheometer method described by Sofra et al. (2007) be adopted. This will enable the full flow diagram to be determined from the vane rheometer tests. The vane rheometer tests should be supplemented with rotary viscometer testing using a bob-and-cup or cone viscometer, and should also be carried out on the full suite of samples.

- Pilot testing must be conducted on samples of representative mineralogy and particle size distribution.

- Pilot testing should be carried out on 'unsheared' as well as fully sheared samples with slump testing to be used in conjunction with laboratory testing to assess the degree of shear thinning of the sample.

When conducting pilot-scale testing, it will be vital to ensure that the $\mathrm{pH}$, flocculant addition and other chemical parameters are known at the time of testing. In fact, as far as is practicable, the pilot-scale testing should be conducted with liquor that is representative of the proposed operation; i.e. testing should proceed after decisions on flocculation type and dose.

Since, other than for operating processing plants, it will be nearly impossible to ensure that the chemistry and the flocculant addition are precisely the same as the full-scale operation, it is suggested that laboratory testing should be used to develop a sensitivity analysis of the estimated yield stress, to variations in $\mathrm{pH}$, flocculant and other chemical parameters. Then, when interpreting the results of the test work, cognisance should be taken of the laboratory-derived sensitivity analysis as well as the sensitivity of the yield stress to testing method.

When comparing laboratory determined flow diagram parameters with those derived from pilot-scale or full-scale trials, cognisance must be taken of the well-known Moody-Rabinowitch correction to derive the bulk shear rates.

\section{Application of rheology to beach profile prediction}

Having ensured that the rheological testing is as representative as possible, all that remains is to select parameters that will enable calculation of the most probable profile. At this stage it is appropriate to take note of the accuracy of the current methods of beach profile estimation. It is the authors' opinion that, based on the current state-of-the-art with regard to beach profile prediction, the statistical uncertainty of the yield stress at the discharge onto the beach, together with the issue of stratification, is likely to introduce uncertainty and variability in the predictions to a similar order of magnitude to the uncertainty associated with the beach profile prediction methods, as they currently stand. It will therefore be vital to conduct a sensitivity analysis and apply probabilistic methods to analysis of the answers, so that profile predictions are presented with confidence bands.

\section{Conclusions}

This paper has documented rheological data measured and collated over a number of mining projects, some of which are greenfields and some of which are in operation. The data presented has shown that the following factors significantly influence the yield stress of a slurry: 
- The testing methodology.

- The $\mathrm{pH}$ of the slurry and by implication the broader chemistry of the slurry.

- The flocculant type and dose.

- The degree of shear thinning (or shear thickening) undergone by the slurry by the time it is discharged onto the beach.

- The extent to which the slurry flow stream stratifies into higher density at the bed as it flows down the beach, which is most likely related to the percentage solids, the flocc characteristics and the degree of shear thinning. It is likely that the sand fraction and the coarseness of this fraction will also have a significant bearing on the density stratification.

The data show that each of the factors listed above can affect the yield stress by between 30 and $300 \%$. It has been demonstrated, using the stream power-entropy beach profile prediction approach, that the yield stress has a significant impact on the beach profile prediction. While yield stress has been the focus of this paper, it should be borne in mind that the full rheological flow diagram has an impact on the beaching predictions and therefore it is important to track the variability of slurry rheology over the full range of shear rates.

It is concluded that it is vital, when carrying out rheological testing and profile prediction that, in addition to ensuring that the slurry is made up of tailings of representative particle size distribution and mineralogy, the slurry must be as representative as possible of the full-scale slurry having regard to the factors listed above. Where practical, rheological tests should be repeated until consistent results are obtained between tests.

When developing beach profile predictions it is vital to carry out a sensitivity analysis and present the results on a probabilistic basis with confidence bands. A rheological testing protocol has been suggested that will assist in the development of the sensitivity analysis and confidence bands.

\section{Acknow ledgements}

The authors would like to acknowledge all mining companies that are braving the bleeding edge of the beach profile prediction technology in their quest to improve the efficiency and effectiveness of tailings management through the adoption of high density tailings. It is these companies that are enabling the accumulation of data and experience that will ultimately mature the technology and benefit the industry as a whole.

\section{References}

McPhail, G. (1995) Prediction of the beaching characteristics of hydraulically placed tailings, PhD Dissertation submitted to the University of the Witwatersrand, Johannesburg, South Africa.

McPhail, G. (2008) Prediction of the Beach Profile of High Density Thickened Tailings from Rheological and Small Scale Trial Deposition Data, Proceedings 11th International Seminar on Paste and Thickened Tailings (Paste08), A.B. Fourie, R.J. Jewell, P. Slatter and A. Paterson (eds), 5-9 May 2008, Kasane, Botswana, Australian Centre for Geomechanics, Perth, pp. 179-188.

Pinto, M. and Barrera, S. (2002) Tailings beach slope forecasting - copper tailings, in Proceedings Ninth International Conference on Tailings and Mine Waste (Tailings and Mine Waste '02) Fort Collins, Colorado, USA, 27-30 January 2002, pp. 87-94.

Simms, P., Williams, M.P.A., Fitton, T. and McPhail, G. (2011) Beaching angles and evolution of stack geometry for thickened tailings - A review, in Proceedings 14th International Seminar on Paste and Thickened Tailings (Paste2011), R.J. Jewell and A.B. Fourie (eds), 5-7 April 2011, Perth, Australia, Australian Centre for Geomechanics, Perth, pp. 323-338.

Sofra, F., Fisher, D.T. and Boger, D.V. (2007) The bucket rheometer for thickened tailings and paste flow curve determination, in Proceedings Tenth International Seminar on Paste and Thickened Tailings (Paste07), A.B. Fourie and R.J. Jewell (eds), 13-15 March 2007, Perth, Australia, Australian Centre for Geomechanics, Perth, pp. 249-258. 
